\title{
Full Length Article
}

\section{Knowledge and attitudes about HIV infection and prevention of mother to child transmission of HIV in an urban, low income community in Durban, South Africa: Perspectives of residents and health care volunteers}

\author{
Firoza Haffejee ${ }^{a, *}$, Katie A. Ports ${ }^{b, 1}$, Maghboeba Mosavel ${ }^{b, 2}$ \\ a Department of Basic Medical Sciences, Durban University of Technology, P.O. Box 1334, Durban 4000, South Africa \\ ${ }^{\mathrm{b}}$ Health Behavior and Policy, Virginia Commonwealth University, PO Box 980149, Richmond, VA 23219, USA
}

\section{A R T I C L E I N F O}

Article history:

Received 18 September 2015

Accepted 9 February 2016

Available online 31 March 2016

Keywords:

AIDS

HIV knowledge

HIV transmission

PMTCT

\begin{abstract}
A B S T R A C T
Background: HIV prevalence is high among South African women of reproductive age and transmission of HIV from mothers to children is a concern. This study ascertained the level of knowledge about HIV infection and prevention, particularly prevention of mother to child transmission (PMTCT) amongst South African women from a low income community. It also established the challenges in delivering HIV education from the perspectives of health care volunteers.

Method: Female residents $(n=67)$ from Kenneth Gardens, a low income community in Durban, South Africa were interviewed. In-depth semi-structured interviews were conducted with 12 health care volunteers who were either health care workers or residents who provided some form of social support in the community.

Results: The majority of respondents indicated that a mother could transmit HIV to her child but were unable to specify how. Many women had general HIV/AIDS knowledge but were unable to identify essential prevention behaviours and were not very receptive to more information on HIV/AIDS. They were supportive of routine testing procedures and child bearing amongst HIV positive women. Health care volunteers indicated a need for a community clinic in the area. They also had limited knowledge of PMTCT and indicated that there was a need for more education on HIV, particularly to encourage the youth and men to use preventative measures. Conclusion: Innovative ways to impart knowledge particularly of PMTCT and updated standards of practice are essential. It is important that the community understands how transmission occurs so that prevention can follow.
\end{abstract}

(c) 2016 The Authors. Publishing services by Elsevier B.V. on behalf of Johannesburg University. This is an open access article under the CC BY-NC-ND license (http://

creativecommons.org/licenses/by-nc-nd/4.0/).

\footnotetext{
* Corresponding author. Tel.: +27 31373 2395; fax: +27 313732405.

E-mail addresses: firozah@dut.ac.za (F. Haffejee), yfb1@cdc.gov (K.A. Ports), mmosavel@vcu.edu (M. Mosavel).

1 Tel.: +1 8046284631 .

2 Tel.: +1 8046282929 .
}

Peer review under responsibility of Johannesburg University. 


\section{Introduction}

South Africa has made strides in reducing the number of new HIV infections through a National Strategic Plan for HIV/AIDS with priorities in expanding access to treatment and support services (Department of Health SA, 2007). However, HIV prevalence continues to be high among women of reproductive age, and transmission of HIV from mother to child (MTCT) is a major concern and remains a public health priority (Department of Health SA, 2007; Ginsburg, Hoblitzelle, Sripipatana, \& Wilfert, 2007; Manzi et al., 2005; UNAIDS, 2008). Children represent more than $15 \%$ of new HIV infections worldwide, and approximately $90 \%$ of children become HIV-positive through MTCT (UNAIDS, 2008). The incidence of HIV infection in children in low and middle income countries (LMICs) in 2012 was 260,000 with 90\% of these in sub-Saharan Africa (UNAIDS, 2013).

Vertical transmission of HIV from mother to child occurs across the placenta or via breast milk (John \& Kreiss, 1996). A more severe maternal infection, indicated by higher viral load and lower CD4 lymphocyte counts, is associated with an increased risk of viral transmission (Lyall et al., 2001). In the absence of any interventions, transmission rates range from 15 to $45 \%$ (World Health Organization, 2014) but can be reduced to below $5 \%$ with effective interventions such as ARVs, caesarean section deliveries and avoidance of breastfeeding (Cooper et al., 2002; Dorenbaum et al., 2002; European Collaborative Study, 2005). However, the avoidance of breastfeeding is not realistic in developing countries and a large proportion of HIV positive women breastfeed their infants due to social pressure (Becquet et al., 2005; Coutsoudis et al., 2001). Thus the guidelines that SA has adopted for prevention of MTCT (PMTCT) include the promotion of exclusive breastfeeding together with the provision of antiretroviral prophylaxis to all neonates born to HIV-positive mothers (Department of Health, 2013). Additionally all HIV-infected pregnant women are provided with ARVs and counselling during pregnancy (Department of Health, 2013).

In 2012 the HIV prevalence among pregnant South African women attending antenatal clinics was $29 \%$, with the highest provincial prevalence of $37 \%$ in KwaZulu-Natal (Department of Health, 2012). According to Goga (2011), one-third of HIVinfected mothers in South Africa received triple-drug antiretroviral therapy (ART) and 20\% reported exclusive breastfeeding, both protective factors for PMTCT. Of concern, is that nearly two-thirds of the pregnancies among HIV-infected women were unplanned and this is a risk factors associated with MTCT (Goga, 2011).

Knowledge of HIV/AIDS and PMTCT has been shown to influence the motivation and uptake of ART for PMTCT among women (Duff, Kipp, Wild, Rubaale, \& Okech-Ojony, 2010). However, previous studies in rural communities in Africa have indicated that low levels of knowledge regarding PMTCT and misconceptions about the cause of HIV are widespread (Asefa \& Beyene, 2013). One of the goals of the PMTCT programme in South Africa is to strengthen community-based activities to enhance the effectiveness of health programmes (Department of Health, 2012). Previous research has indicated that such community-based activities have been established in the current study setting, however the level of knowledge particularly around HIV and PMTCT of these health care volunteers is unknown.

The aim of this study was to ascertain the level of knowledge and attitudes about HIV infection and prevention particularly PMTCT of HIV amongst South African women residing in Kenneth Gardens, Durban. The study also aimed to understand the key challenges in delivering health care services and HIV education from the perspectives of community health care volunteers.

\section{Method}

\subsection{Study population and sampling}

This is a collaborative study between researchers at the Durban University of Technology (DUT) and Virginia Commonwealth University (VCU). Researchers from DUT have been working closely with the Kenneth Gardens community in health and community development projects. Data presented in this paper are a sub-section of a larger study on perceptions of health care particularly regarding HIV and cervical cancer. Permission to conduct the study was obtained from the Institutional Research Ethics Committee (IREC) of DUT and the Institutional Review Board for the Protection of Human Subjects at VCU. Written informed consent was obtained from all participants prior to data collection, between July and September 2013.

Kenneth Gardens is a municipal housing estate, comprising a low income community, within the large metropolitan city of Durban. It offers subsidised housing to approximately 1500-1800 people of a low socio-economic income or with a mental or physical disability. Participants comprised 67 women who are residents in Kenneth Gardens and 12 health care volunteers who were either health care workers or residents who provided some form of service or social support in the community. The health care volunteers were identified by members of the DUT research team through previously established collaborative programmes between DUT and the community. Health care volunteers assisted in participant recruitment by word of mouth. Residents were further recruited using a snowball technique which is effective when recruiting people from a specific population, in this case from Kenneth Gardens only. In order to reduce selection bias, and ensure that participants were members of different social groups, all health care volunteers were asked to help recruit women. Moreover, we had to consider the safety of our research team. As a team of women we were advised not to go door to door, but to have community members come to us in a central location.

\subsection{Data collection and management}

A mixed methods approach was used, whereby both openand closed-ended questions were part of the questionnaire. 
The closed-ended questions allowed the researchers to obtain quantitative data to answer specific research questions. The open ended responses enabled more in-depth understanding of the participants' knowledge, attitudes, perceptions and behaviour related to HIV. Data collected for this paper were part of a larger study investigating women's health in Kenneth Gardens. Details of data collection and interviews have been described previously (Ports, Haffejee, Mosavel, \& Rameshbabu, 2015).

\subsubsection{Interviews with residents}

Study inclusion criteria included being a female resident of Kenneth Gardens, between the ages 18-50, with no mental disability and had to be fluent in either English or isizulu. The informed consent was reviewed aloud with all participants, in the participants' language of choice. A trained interviewer administered the structured questionnaire. Interviews were recorded via paper and pencil on the survey document. The isiZulu interviews were conducted by two trained isizulu speaking research assistants. Researchers (FH and KP) conducted the English interviews with residents. All interviews were conducted in a private room following informed consent. As previously noted (Ports et al., 2015), the structured interview consisted of 133 questions and took approximately one hour to complete. However, only questions pertaining to HIV were analysed for the present paper. HIV positive women answered 96 questions whilst HIV negative women answered 67 questions relevant to this report. This included the demographic and general health related questions. The remaining questions were related to cervical cancer and this has been reported previously (Ports et al., 2015).

On completion of the interview, all participants received an honorarium of R135.00 (equivalent to \$15). The study adhered to the principles of the Declaration of Helsinki (World Medical Association, 2013).

\subsubsection{Health care volunteer interviews}

Study inclusion criteria included being a female who provided health related assistance to the residents of Kenneth Gardens. All health care volunteers had to be fluent in English. Following informed consent, the semi-structured interviews were conducted in a private room in English by KP and took approximately $45 \mathrm{~min}$ to complete. These interviews were audio-recorded and transcribed. The interview guide included 16 open-ended questions, but only questions pertaining to informants' perceptions about the community's HIV knowledge and attitudes were analysed for the purpose of this paper. Sample questions included: "What challenges do women in Kenneth Gardens experience when accessing HIV screening and treatment?" and "What do people in your community know about mother to child transmission of HIV?"

On completion of the interview, all these participants also received an honorarium of R135.00 (equivalent to $\$ 15$ ).

\subsection{Data analysis}

SPSS (version 17) was used to analyse quantitative data. Utilising a thematic approach, qualitative data were analysed by identifying recurring themes (Braun \& Clarke, 2006). Two coders (KP and a trained research assistant) independently reviewed the transcripts of the qualitative responses and developed a list of preliminary codes.

\section{Results}

\subsection{Residents}

\subsubsection{Socio-demographic characteristics of residents}

A total of 67 residents were interviewed. The mean age was 28.4 years (18-50). Most were identified as Black (93\%; $n=62), 4 \%(n=3)$ Coloured and $3 \%(n=2)$ of Indian origin. This racial profile is reflective of the population diversity in KG (Erwin, Marks, \& Couchman, 2014). The majority of respondents were single (77\%; $n=52)$, had completed high school (43\%; $n=29)$ and were currently unemployed $(72 \%$; $n=48)$. Approximately $22 \%(n=15)$ were HIV positive. Detailed socio-demographic data have been provided previously (Ports et al., 2015).

\subsubsection{HIV/AIDS knowledge}

Many respondents were unable to distinguish the difference between HIV and AIDS with 82.1\% $(n=55)$ reporting that HIV and AIDS were synonymous. The majority of respondents were knowledgeable about the contraction of HIV from unprotected sex and the sharing of needles or razor blades with infected people. However there was a misconception about the spread of HIV via mosquito bites, oral sex, coughing, sneezing, sharing of utensils and shaking hands with HIV infected people (Table 1). They agreed that sex with multiple partners could increase the risk of contracting HIV (94\%; $n=63$ ) and also agreed that use of a condom during sex lowers the chance of contracting HIV $(91 \% ; n=61)$. They were also aware $(91 \% ; n=61)$ that people with other sexually transmitted diseases are at greater risk of HIV. There were misconceptions about the prevention of HIV/AIDS by seeking the help of a traditional healer and by the washing of genitals after sexual intercourse (Table 1$)$.

Only $67.2 \%(n=45)$ respondents were aware that ART can prevent the development of AIDS in an HIV infected person. However, $98.5 \%$ correctly reported that people with HIV could prolong their lives by taking medications. However, a

\section{Table 1 - HIV/AIDs knowledge and awareness.}

Confirming responses to contraction of HIV by:

Unprotected sex $97(65)$

Sharing of needles

$95.5(64)$

Sharing of razor blades $\quad 97(65)$

Mosquito bites $31.3(21)$

Oral sex

$63(41)$

Sharing of utensils with HIV infected people 11.9 (8)

Coughing and sneezing

$13.4(9)$

Shaking hands with an HIV infected person $3(2)$

By a curse 6 (4)

Confirming responses to prevent getting HIV/AIDS, by:

A traditional healer

Bathing/washing genitals after intercourse 
misconception existed amongst $35.8 \%(n=24)$ of respondents that medicine was available to completely cure an HIV infection.

\subsubsection{Mother to child transmission}

The majority of respondents $(91 \% ; n=61)$ indicated that a mother could transmit HIV to her child. However, when asked to describe how MTCT occurred, women seemed less certain; slightly over half of the women $(n=37 ; 55 \%)$ specified that the virus could be transmitted during the pregnancy, less $(n=18$; $27 \%$ ) reported transmission during labour/delivery, and only 19 (29\%) indicated transmission through breastfeeding. Some respondents ( $n=14 ; 21 \%$ ) indicated that the virus could be transmitted if the mother "failed to take her pills." A few suggested that transmission could occur if the mother engaged in risky sexual behaviours such as unprotected sex during the pregnancy $(n=3)$.

The majority of respondents $(94 \%, n=63)$ reported that a pregnant woman who was HIV positive needed to continue to practice safe sex despite being pregnant. Many $(52 \%, n=35)$ described the need to use a condom as a way to protect the baby. Other respondents $(24 \%, n=16)$ noted that HIV positive women always need to wear a condom in order to maintain their viral load. Fewer respondents suggested that condoms should be worn to protect the sexual partner $(12 \%, n=8)$, and to protect the women from other STIs $(7 \%, n=5)$. Respondents $(6 \%, n=4)$ who did not think it necessary to use a condom indicated that it was unnecessary, because the woman was already infected; "you're already infected, what difference does it make?"

3.1.4. Attitude towards child bearing for HIV positive women Two thirds of the respondents $(n=45 ; 67 \%)$ indicated that it was acceptable for an HIV infected woman to conceive. Some participants expressed the importance of child bearing to the role of women in relationships $(19 \%, n=13)$. "It is a principle that every woman should fall pregnant" and "She needs to feel like a woman - feel normal". Others indicated that if an infected woman took her medication $(12 \%, n=8)$, was healthy $(10 \%, n=7)$, had a high CD4 count $(10 \%, n=7)$, planned with her doctor $(7 \%, n=5)$ and her partner $(4 \%, n=3)$ then she should be able to conceive: "It depends if you are healthy, have a high CD4 count plus you take your medicine, it's OK. Your life should not end if you get HIV." However a minority believed that women should not conceive and expressed that it endangered the child $(7 \%, n=5)$, and the pregnant woman herself $(13 \% ; n=9)$, especially during the delivery.

\subsubsection{Attitudes towards screening for HIV}

Two thirds of the respondents $(n=45 ; 67 \%)$ indicated that they were aware of routine HIV testing, where patients are tested for HIV during routine medical visits. All respondents were read a brief description of routine testing, and were asked their opinion about it. The majority $(94 \% ; n=63)$ felt that routine testing was a good idea because it was important to know their status $(54 \% ; n=36)$. One woman mentioned, "Everyone should know their status. We're all positive until proven negative." Another added, "One may have a disease without realizing that one is sick due to being infected". Ten participants (15\%) mentioned that routine screening was particularly important, because of the HIV infection window period. Others $(9 \%, n=6)$ thought that routine testing must be made mandatory; "If left up to us, we won't do it." Some $(10 \% ; n=7)$ commented that frequent testing could help with HIV infection control, i.e., maintenance of viral load and early diagnosis. Few respondents $(6 \%, \mathrm{n}=4)$ indicated that routine testing should not be mandatory and felt that it had to be a personal choice; "It has to be a choice, some people are not ready for it. Some don't want to know."

\subsubsection{Delivering HIV/AIDS information}

The majority of respondents $(73 \%, n=49)$ indicated that they had enough information about HIV/AIDS and that they did not need more information. Fewer woman $(27 \%, n=18)$ reported that they did not have sufficient information.

The respondents indicated that effective ways to disseminate HIV/AIDS information to women in their community would be via television programmes $(31 \%, n=21)$, health care workers $(24 \% n=16)$, newspaper/magazines $(18 \%, n=12)$, other print materials $(12 \%, n=8)$, teachers/schools $(7 \%, n=5)$, internet/social networks $(6 \%, n=4)$ and radio $(6 \%, n=4)$. They also $(16 \%, n=11)$ indicated that having community workshops/seminars would be a good way to distribute information. Others ( $5 \% ; n=3$ ) suggested going door-to-door for dissemination of information.

When asked to provide their thoughts on ways to improve HIV prevention, some respondents indicated that more education was needed $(25 \%, n=17)$, free condoms should be distributed regularly $(22 \%, n=15)$, emphasis should be placed on using protection during sex $(13 \%, n=9)$. Respondents indicated that community workshops would be ideal if these targeted the youth and men and if the workshops had HIV infected people discussing issues that affected them.

\subsubsection{HIV testing, medication and disclosure}

The majority of respondents $(90 \%, n=60)$ reported having had an HIV test. Of these, $50 \%(n=33)$ were tested within the last year. The prevalence of HIV amongst the study population was $22.4 \%(n=15)$.

The majority of HIV infected participants $(66.7 \%, n=10)$ were on ART and only one of these indicated that she was negligent in taking her full course of medication. Three respondents (5\%) reported that their CD4 $\mathrm{T}$ cell count is above the required level for receiving ART. All the women on ART reported that the medication had a positive effect on their health.

Only one respondent reported that she had not disclosed her status to anyone whilst two respondents (3\%) indicated disclosure to everyone that they knew. The majority of respondents disclosed their status to a family member, typically the mother $(80 \% ; n=12)$ and/or their current partner $(66.7 \% ; n=10)$.

\subsubsection{Attitudes towards women who had disclosed their HIV} status

Of the 15 respondents who indicated that they were HIV positive, two (13\%) indicated that they had been treated badly by a health worker because of their status. Two (13\%) respondents also reported losing their job, one indicated that she had been denied care by her family, one had been rejected by her family 
and another reported that she had been treated badly at work/ school. Some respondents indicated that they had been abused by their partners because of their status $(20 \% ; n=3)$ and another $20 \%(n=3)$ had experienced a break-up of their relationship because of their status. Some respondents reported losing their friends because of their status $(26.7 \% ; n=4)$.

Personal concerns reported by HIV infected participants were worries about death and leaving their children behind $(33 \% ; n=5)$. They also commented that they worried about becoming sick when their CD4 T cell count dropped (20\%; $n=3)$ and are concerned about how others treat them (13\%; $n=2)$. A further two respondents (13\%) expressed concern over the treatment; taking ART for the rest of their lives, and actually getting the ART. One was concerned about how she contracted HIV.

\subsection{Perspectives of health care volunteers}

\subsubsection{Socio-demographic characteristics of health care volunteers}

A total of 12 female health care volunteers were interviewed. The mean age was 56 years (50-63). Majority were identified as Black $(83 \%, n=10)$ and $17 \%(n=2)$ were White. Three $(25 \%)$ were single, five (42\%) were married and four (33\%) widowed. Only three $(25 \%)$ of the health care volunteers had some form of health related qualification (Homoeopath: $\mathrm{n}=1$, Nurse: $\mathrm{n}=1$, Diploma in Occupational Therapy and Reflexology: $\mathrm{n}=1), 50 \%(\mathrm{n}=6)$ had completed high school while $25 \%(\mathrm{n}=3)$ had not completed high school. Only one health care volunteer was a paid staff member affiliated to the Homeopathy clinic. The remaining 11 were informal helpers who either provided assistance to the staff at the Homeopathy clinic or advocated for the health and well-being of fellow residents by providing help in the form of ensuring that the sick take their medications regularly, bathing the frail or providing some form of health advice. The majority were HIV negative $(92 \%$, $\mathrm{n}=1$ ) and only one (8\%) was HIV positive.

\subsubsection{Stigma}

Health care volunteers indicated that whilst there was much gossip about people who were sero-positive, these people were well cared for by the community and that they were assisted in times of need. One health care volunteer however, indicated that health care professionals such as doctors and nurses in the public sector hospitals were insensitive towards HIV infected patients and this led to patients 'losing hope'. However there seemed to be more sensitivity towards children who were infected by the virus; for example some parents avoided revealing to their own children about another child's status. So in this way infected children were not ostracised.

\subsubsection{Challenges}

Health care volunteers reported that a major challenge in accessing health care was the long distance to clinics $(50 \% ; n=6)$, the lack of personal transport $(25 \% ; n=3)$ and insufficient funds $(17 \% ; n=2)$ to use public transport to access facilities. They indicated a need for a community clinic in the area. Some of these respondents also indicated that the lack of nutritious food $(8 \% ; n=1)$ and warm clothing during winter $(8 \% ; n=1)$ were further challenges experienced by the sick as this prolonged illnesses especially TB which often accompanied an HIV infection.

\subsubsection{Condom usage}

The health care volunteers were aware of the low uptake in the use of condoms. 'South African men don't like them ... they don't wear them and the men like dry sex so then it's rough'. Another health care volunteer mentioned that she tried to encourage young girls to use condoms but after a few months of dating they stopped using them because the male partner felt that he had to be trusted. These girls were however aware of the male partner being promiscuous.

\subsubsection{PMTCT knowledge}

The health care volunteers mentioned that the community was aware of HIV but they felt that there was limited knowledge of MTCT. Furthermore they themselves had limited knowledge of MTCT and only three (25\%) health care volunteers indicated that MTCT could occur via breastfeeding and one (8\%) of these women indicated that HIV infected mothers are told to stop breastfeeding. Health care volunteers mentioned that women have difficulty abstaining from breastfeeding their children, because of the stigma associated with not being able to care for one's child, and the difficulty in obtaining formula. Another health care volunteer had a vague idea of some link between MTCT and breastfeeding but was uncertain about what advice to give mothers regarding this. She indicated that mothers would need to be referred to medical doctors for advice. Two health care volunteers $(17 \%)$ mentioned that ARVs play a role in PMTCT. However, one of these informants mentioned that HIV infected women often get pregnant in order to receive ARVs because of the belief that the ARVs will cure them of HIV but they were unaware that the ARVs are actually for PMTCT.

\subsubsection{HIV education}

The health care volunteers indicated that there was a need for more education on HIV, particularly to encourage the youth and men to use preventative measures. They however indicated that people were unwilling to attend talks as they found this 'boring' and thus innovative ways of delivering information were required. These could be plays or movies about HIV and enticing people to attend by the provision of food, snacks or even gifts such as 'hand cream'. They indicated that this would serve to draw people to the information session as many were unable to afford bare necessities.

\section{Discussion}

This study highlights the complex interplay of many factors involved in HIV knowledge and prevention among women residing in an urban community of low socio-economic standing. Although there is knowledge about HIV/AIDS transmission modes and prevention strategies, there are many misconceptions regarding the modes of transmission. There is a general indication of awareness of MTCT, but difficulty in specifying how it occurs. Of concern is that the health care volunteers have limited PMTCT knowledge and this would be related to their lack of training in a health related field. Whilst some of these health care volunteers 
indicated that HIV infected mothers were told to stop breastfeeding, none of them correctly indicated that the virus could be present in breast milk. They were also unaware that in the African context, the current measure is to promote exclusive breastfeeding as opposed to mixed feeding (Department of Health, 2013). The rate of MTCT is higher in babies who receive both breast milk and formula compared to those who are exclusively breastfed (Iliff et al., 2005). Mixed feeding is associated with less maternal antibodies due to consumption of less breast milk. Additionally, contaminated fluids in mixed fed babies may damage the bowel, impair mucosal integrity and facilitate the entry of the virus into infant tissues from the breast milk (Coutsoudis et al., 2001).

Because HIV infections are high among women during the reproductive years, it is important that they understand MTCT of HIV which is a growing problem in resource-poor settings where there is an estimated risk of $21 \%-45 \%$ (Maputle \& Jali, 2008; Simonsen et al., 2014). Our findings are consistent with those of Maputle and Jali (2008) who also reported that women have limited knowledge of HIV transmission through breastfeeding. A further concern that the present study highlights, is that ARVs during pregnancy are for curing the mother and not for PMTCT. Educational programmes highlighting PMTCT are thus required to dispel misconceptions around these issues. In order for PMTCT initiatives to be successful, creative ways of disseminating this information is important.

The general acceptability of childbearing by HIV positive women is consistent with previous reports (Maputle \& Jali, 2008). Positive attitudes regarding conception of HIV positive women were based on the fact that HIV transmission to the child could be prevented through proper planning with their partners, the medical doctor, taking ART to prevent transmission of the virus to the baby and to boost CD4 cell counts. There is however also a myth about condom use during pregnancy being essential to prevent vertical transmission. There were fewer responses that condom usage is for protection of the partner and for prevention of other sexually transmitted infections during pregnancy further highlighting the need for HIV educational programmes in this community.

Whilst the knowledge of HIV transmission via unprotected sexual activity is high, it is disturbing that only $10 \%$ of the population use condoms. This, together with the reported large number of sexual partners is in stark contrast with their knowledge that multiple partners increase the risk of contracting HIV. Clearly some form of intervention is required to encourage people to reduce their number of sexual partners and to use condoms. Women however experience difficulty in negotiating safe sex. Furthermore health care volunteers mentioned that men don't like to use condoms because men like 'dry sex'. This seems to be achieved by very little foreplay so that the vagina remains dry and females also allegedly use traditional herbs for drying out the vagina. This is cause for concern as this could lead to easy tearing of tissues and increased exposure to HIV. While our study contributes important information regarding particular knowledge and attitudes of a specific community in order to tailor interventions appropriately, additional research focused on issues pertaining to challenges that individuals face when adopting recommended health behaviours, such as condom use, is needed. It is likely that issues including gender(in) equity, and notions of male masculinity impact on what women are able to do with the information that they receive (Leddy et al., 2015). Thus, it is imperative that we not only know how to provide appropriate information, but also the broader context that supports the adoption of healthier behaviours.

Many of the women thought that they have enough information about HIV/AIDS; however some are still willing to obtain more information. This creates an opportunity for establishing and enhancing HIV educational programmes that are specific for small communities. Some of the options raised included: mobilising women to go door to door giving information, conducting community workshops in the form of 'movies' and the provision of free food to draw people to such events. It must be noted that this is a low income community and like many others in South Africa, people live below the bread line, and thus, incentives were seen as an important means to bolster participation.

Although HIV treatment in countries with limited resources, is expanding, there is a concern of poor adherence which could lead to viral resistance to the medication (Bekker, Myer, Orrell, Lawn, \& Wood, 2006). Conversely, the majority of HIV positive women in this study adhere to their medication and they are sure that it helps improve their lives. Those who are not on medication knew that their CD4 cell count was too high to receive ARVs. In developing countries including South Africa, HIV positive people only qualify for treatment once their CD4 cell count has dropped to below 350 cells/ $\mu$ l (Bekker et al., 2006). Although it was only one case, where a woman reported to not rely on HIV treatment, but on God, this highlights the need for the presence of knowledgeable religious and other support groups who could simultaneously encourage their constituents to adhere to health behaviours.

The majority of the women in this study had disclosed their HIV status, particularly to mothers and current partners. Studies have reported that HIV disclosure comes with different sorts of stigma and discrimination (Simoni, Demas, Mason, Drossman, \& Davis, 1998). We report that women face bad treatment from health care workers, family, partners and society. These factors could make people more secretive about their HIV status, a further challenge in the fight against HIV. More work is needed to impart information to communities and health services so that individuals can be sensitive to HIV, and so that patients have more positive health care experiences. Although the majority of women seemed optimistic about their lives despite the HIV infection, there are still concerns about becoming ill, death and leaving their children behind. These concerns highlight that women need advice and support that will help them cope with their HIV status.

Given the high levels of poverty and the distance to the health facility and transport problems, there is a need for community based care. However, this study highlights that there is an urgent need to provide the relevant education to health care volunteers prior to them taking on this role. Without this education, incorrect information will be passed on by care givers to the community. Short courses that provide the relevant training are thus essential. Refresher courses for qualified health care workers are also crucial so that they can continue with the most updated information. 
While this study provides important information for HIV prevention efforts, it is not without limitation. For example, this study utilized a snowball sampling technique, which does not provide a random, unbiased sample, which could have led to more popular individuals in the community being more likely to be sampled. However, these individuals are also more likely to be active members of their community, and thus, it is particularly important to tap into their perceptions about health care delivery as they are often an important conduit for providing information about health and services. In addition, the study only included the perceptions and experiences of women from Kenneth Gardens and may not be generalizable to other settings. Moreover, only women were included in this study. It would be useful to understand the perceptions and experiences of men in the community, as well as care providers from nearby hospitals, and political figures.

\section{Conclusion}

Given that women reported that they are knowledgeable about HIV/AIDs, but were unable to identify how to prevent transmission from mother to child, innovative ways to impart knowledge and updated standards of practice are essential. A practical solution to impart PMTCT knowledge could be to provide training to the health care volunteers, who could then provide tailored training to their community. In addition, the Homeopathic clinic could also provide PMTCT guidance during their routine exams. However, more research is needed to understand how to best tailor this information so that the community is receptive to receiving it.

\section{Acknowledgements}

This study was supported by funding from NIH R25CA093423 and the International Partnerships Major Initiative Award from Virginia Commonwealth University, Richmond, Virginia USA.

\section{R E F E R E N C E S}

Asefa, A., \& Beyene, H. (2013). Awareness and knowledge on timing of mother-to-child transmission of HIV among antenatal care attending women in Southern Ethiopia: a cross sectional study. Reproductive Health, 10. doi:Artn 66. doi 10.1186/1742-4755-10-66.

Becquet, R., Ekouevi, D. K., Viho, I., Sakarovitch, C., Toure, H., Castetbon, K., ..., \& Leroy, V. (2005). Acceptability of exclusive breast-feeding with early cessation to prevent HIV transmission through breast milk, ANRS 1201/1202 Ditrame Plus, Abidjan, Cote d'Ivoire. Journal of Acquired Immune Deficiency Syndromes, 40(5), 600-608. doi:00126334-20051215000015 [pii].

Bekker, L. G., Myer, L., Orrell, C., Lawn, S., \& Wood, R. (2006). Rapid scale-up of a community-based HIV treatment service: programme performance over 3 consecutive years in Guguletu, South Africa. S Afr Med J, 96(4), 315-320. Retrieved from: http://www.ncbi.nlm.nih.gov/pubmed/ 16670804.
Braun, V., \& Clarke, V. (2006). Using thematic analysis in psychology. Qualitative Research in Psychology, 3, 77-101. http:// dx.doi.org/10.1191/1478088706qp063oa.

Cooper, E. R., Charurat, M., Mofenson, L., Hanson, I. C., Pitt, J., Diaz, C., ..., \& Blattner, W. (2002). Combination antiretroviral strategies for the treatment of pregnant HIV-1-infected women and prevention of perinatal HIV-1 transmission. Journal of Acquired Immune Deficiency Syndromes, 29(5), 484-494.

Coutsoudis, A., Pillay, K., Kuhn, L., Spooner, E., Tsai, W. Y., \& Coovadia, H. M. (2001). Method of feeding and transmission of HIV-1 from mothers to children by 15 months of age: prospective cohort study from Durban, South Africa. AIDS, 15(3), 379-387. Retrieved from: http://www.ncbi.nlm.nih.gov/ entrez/query.fcgi?

$\mathrm{cmd}=$ Retrieve $\& \mathrm{db}=$ PubMed\&dopt=Citation\&list_ uids $=11273218$.

Department of Health. (2012). Saving mother's report. Retrieved from: http://www.doh.gov.za/docs/reports/2012/ savingmothersshort.pdf.

Department of Health. (2013). The South African antiretroviral treatment guidelines version 14. Retrieved from South Africa.

Department of Health SA. (2007). HIV and AIDS and STI strategic plan for South Africa 2007-2011.

Dorenbaum, A., Cunningham, C. K., Gelber, R. D., Culnane, M., Mofenson, L., Britto, P., ..., \& Sullivan, J. L. (2002). Two-dose intrapartum/newborn nevirapine and standard antiretroviral therapy to reduce perinatal HIV transmission: a randomized trial. JAMA, 288(2), 189-198.

Duff, P., Kipp, W., Wild, T. C., Rubaale, T., \& Okech-Ojony, J. (2010) Barriers to accessing highly active antiretroviral therapy by HIV-positive women attending an antenatal clinic in a regional hospital in western Uganda. Journal of the International AIDS Society, 13, 37.

Erwin, K., Marks, M., \& Couchman, I. (2014). Homeopathic health care in a low-income housing estate in Durban: possibilities for a plural health care model in South Africa. The International Journal of Health Wellness and Society, 3(3), 1-16.

European Collaborative Study. (2005). Mother-to-child transmission of HIV infection in the era of highly active antiretroviral therapy (Retrieved from).

Ginsburg, A. S., Hoblitzelle, C. W., Sripipatana, T. L., \& Wilfert, C. M. (2007). Provision of care following prevention of mother-to-child HIV transmission services in resource-limited settings. AIDS, 21(18), 2529-2532.

Goga, A. (2011). Impact of the national prevention of mother to child transmission (PMTCT) program on mother-to-child transmission of HIV (MTCT), South Africa, 2010. In Paper presented at the 6th international AIDS society conference, Rome.

Iliff, P. J., Piwoz, E. G., Tavengwa, N. V., Zunguza, C. D., Marinda, E. T., Nathoo, K. J., ..., \& Humphrey, J. H. (2005). Early exclusive breastfeeding reduces the risk of postnatal HIV-1 transmission and increases HIV-free survival. AIDS, 19(7), 699-708. doi:00002030-200504290-00007 [pii].

John, G. C., \& Kreiss, J. (1996). Mother-to-child transmission of human immunodeficiency virus type 1. Epidemiologic Reviews, 18(2), 149-157.

Leddy, A., Chakravarty, D., Dladla, S., de Bruyn, G., \& Darbes, L. (2015). Sexual communication self-efficacy, hegemonic masculine norms and condom use among heterosexual couples in South Africa. AIDS Care, 28(2), 228-233.

Lyall, E. G., Blott, M., de Ruiter, A., Hawkins, D., Mercy, D., Mitchla, Z., ..., \& Taylor, G. P. (2001). Guidelines for the management of HIV infection in pregnant women and the prevention of mother-to-child transmission. HIV Medicine, 2(4), 314-334.

Manzi, M., Zachariah, R., Teck, R., Buhendwa, L., Kazima, J., Bakali, E., ..., \& Humblet, P. (2005). High acceptability of voluntary counseling and HIV-testing but unacceptable loss to 
follow-up in a prevention of mother-to-child HIV

transmission programme in rural Malawi: scaling-up requires a different way of acting. Tropical Medicine \& International Health, 10(12), 1242-1250.

Maputle, M. S., \& Jali, M. N. (2008). Pregnant women's knowledge about mother-to-child transmission (MTCT) of HIV infection through breast feeding. Curationis, 31(1), 45-51. Retrieved from: http://www.ncbi.nlm.nih.gov/ pubmed/18592948.

Ports, K. A., Haffejee, F., Mosavel, M., \& Rameshbabu, A. (2015). Integrating cervical cancer prevention initiatives with HIV care in resource-constrained settings: a formative study in Durban, South Africa. Global Public Health, 1-14. http:// dx.doi.org/10.1080/17441692.2015.1008021.

Simoni, J. M., Demas, P., Mason, H. R. C., Drossman, J. A., \& Davis, M. L. (1998). HIV disclosure among women of African descent: associations with coping, social support, and psychological adaptation. AIDS and Behavior, 4(4), 147-158.
Simonsen, S. E., Kepka, D., Thompson, J., Warner, E. L., Snyder, M., \& Ries, K. M. (2014). Preventive health care among HIV positive women in a Utah HIV/AIDS clinic: a retrospective cohort study. BMC Womens Health, 14(1), 37. http://dx.doi.org/ 10.1186/1472-6874-14-37.

UNAIDS. (2008). United nations joint program on HIV/AIDS. Report on the global AIDS epidemic.

UNAIDS. (2013). Report on the global AIDS epidemic, Geneva, joint united nations programme on HIV/AIDS.

World Health Organization. (2014). Elimination of mother-to-child transmission (EMTCT) of HIV and syphilis Global guidance on criteria and processes for validation. Retrieved from: http://www. who.int/hiv/pub/emtct-validation-guidance/en/.

World Medical Association. (2013). World medical association declaration of Helsinki: ethical principles for medical research involving human subjects. JAMA, 310(20), 2191-2194. http:// dx.doi.org/10.1001/jama.2013.281053. 